\title{
Des enseignants du primaire parlent de la géographie et de son enseignement
}

\section{Samuel Fierz}

Qu'enseigne-t-on en géographie à l'heure actuelle et comment s'y prend-on? Alors que l'on pourrait s'attendre à une très grande diversité de pratiques, l'analyse d'entretiens semi-directifs réalisés en 2001 avec six enseignant(e)s valaisan(ne)s de 5 e et Ge primaire atteste d'habitudes scolaires bien établies. C'est généralement une démarche frontale améliorée qui est mise en auvre; les savoirs sont plus ou moins longuement explicités avant d'être institutionnalisés; les connaissances descriptives sont prédominantes par rapport aux savoir-faire et habiletés intellectuelles, même si l'on veut aussi les développer; la convergence des pratiques autour de ces habitudes est en partie liée à une certaine conception de l'évaluation. Ces traditions disciplinaires sont mises en évidence grâce aux "mondes d'éducation" (Chatel, 1997), un outil d'analyse de l'action éducative qui se noue en classe entre enseignant, élèves et savoirs.

\section{Introduction}

Le courant de recherche francophone en didactiques des sciences sociales (en France, Audigier et l'Institut national de recherche pédagogique [INRP], l'équipe de Le Roux à Caen, Masson à Grenoble, ainsi que l'Association française pour le développement de la géographie [AFDG]; Laurin au Canada; Partoune et Mérenne-Schoumaker en Belgique; etc.) est traversé d'interrogations insistantes sur ce qui s'enseigne et ce qui s'apprend en géographie à l'heure où le monde et les discours sur le monde sont si diversifiés. Notre recherche s'inscrit dans ce courant, et plus précisément dans la lignée des travaux d'Audigier (1999); elle analyse les discours que des enseignants ${ }^{1}$ de l'école primaire ont sur la géographie et son enseignement.

Le présent article est un condensé d'une recherche plus étendue (Fierz, 2002); il s'articule en quatre parties: cadre conceptuel, dispositif de recherche, analyse des données et résultats, conclusions d'ordres théorique, méthodologique et pratique. 


\section{Cadre conceptuel}

L'enseignement de la géographie est ici appréhendé au travers de deux conceptsclés: la discipline scolaire et les mondes d'éducation. Le concept de discipline scolaire pose le cadre de l'action enseignante alors que les mondes d'éducation sont convoqués en tant qu'instrument pour analyser les discours sur l'action.

\section{La géographie en tant que discipline scolaire}

En didactique de la géographie, deux manières de penser les relations entre les savoirs scolaires et ses références, notamment scientifiques, ont été explorées. L'une cherche à identifier des filiations - plus ou moins reconstruites - avec les savoirs savants; elle est souvent assimilée au processus de transposition didactique. Se fondant sur le concept de discipline scolaire, l'autre approche renverse le raisonnement et considère les savoirs scolaires comme une création originale de l'école, qui, selon ses propres règles et finalités, construit ses objets et méthodes d'enseignement; dans ce processus créatif, l'école effectue des emprunts à des sources hétéroclites dont, occasionnellement, les savoirs savants. Cette deuxième perspective constitue un cadre fécond pour notre problématique, car le milieu scolaire y est pensé comme un lieu d'initiative.

Telle que théorisée par Chervel (1988), la discipline scolaire présente quatre caractéristiques majeures. Elle repose avant tout sur une vulgate, c'est-à-dire un ensemble de savoirs partagés, de contenus d'enseignement. À ces contenus sont associés un certain nombre d'exercices typiques qui les mettent en scène. S'ajoutent à cela des procédures d'évaluation standardisées ainsi que des stratégies rodées de motivation et d'incitation au travail. Le concept de discipline scolaire est étroitement lié aux finalités, notamment aux finalités culturelles et éducatives.

Pour ce qui est de la géographie scolaire proprement dite, Audigier (1995) en a formalisé les contours sur la base d'une analyse des textes officiels français pour l'enseignement secondaire. En étudiant les finalités, les contenus et les méthodes, il construit un modèle disciplinaire classique qui présente quatre caractéristiques majeures que nous reformulons ici.

1. On enseigne les résultats, c'est-à-dire ce que l'on sait sur un objet ou ce que l'on tient aujourd'hui pour vrai, et l'on occulte généralement la construction de ce savoir.

2. On évacue les débats pour ne prendre que des références consensuelles.

3. On refuse le politique et les enjeux idéologiques ou éthiques autour du savoir; tout savoir se justifie par sa scientificité.

4. Comme on n'enseigne que les résultats et non leur construction, on se trouve dans un réalisme où tout ce qui est vu, observé ou lu est la réalité (et est donc vrai); cartes, photos, observations ou textes ne sont pas considérés comme des constructions intellectuelles.

Ce modèle que l'auteur français qualifie de «républicain» est fortement marqué par les finalités patrimoniales ou civiques que l'on confere généralement à la géo- 
graphie et à l'histoire, disciplines chargées de transmettre une représentation partagée du territoire et de la mémoire. Audigier (1995) souligne que ce modèle est fortement remis en question depuis les années septante. Il observe un glissement vers un modèle «boîte à outils» orienté davantage par des finalités d'instrumentation: il faut que l'élève acquière un certain nombre de repères spatio-temporels, de notions et de concepts qui sont assortis de quelques éléments de méthode sensés favoriser leur transfert à d'autres thèmes.

L'auteur analyse les raisons de ces changements et en formule quatre. Tout d'abord, le territoire n'est plus à défendre. Ensuite, il devient de plus en plus difficile de construire une référence partagée à l'heure où la mondialisation est en marche et où la société et les publics scolaires sont très diversifiés. De plus, face à cette diversité, la demande par rapport à l'école a changé; il convient désormais de respecter les différences culturelles (droit à l'identité) tout en s'efforçant d'estomper les différences d'origine sociale (droit à l'égalité). Enfin, les savoirs scientifiques ont pris des développements très variés rendant encore plus délicate la question de savoir ce qu'il faut enseigner.

\section{Rendre compte de L'action enseignante}

Les développements de la recherche en didactique ont montré qu'on ne peut pas considérer l'enseignant comme un applicateur des programmes mais bien comme un acteur. Le concept d'action formalise cette approche en permettant de penser ce qui se passe en classe entre l'enseignant et les élèves comme un jeu d'acteur, où chacun a des intentions (notamment sur les savoirs), analyse les situations, prend des décisions et pose des actes. L'action enseignante n'est donc pas entièrement prévisible; elle relève d'une négociation. C'est dans cette perspective que Chatel (1997) développe le concept des mondes d'éducation. Il s'agit d'un modèle d'analyse qui permet de saisir la diversité de l'action éducative par une catégorisation en quatre «mondes». Cet instrument a été développé pour travailler sur des observations menées en classe et a également été utilisé pour analyser les résultats de l'action éducative, à savoir des évaluations et leur notation; dans cette recherche, nous faisons l'hypothèse méthodologique que son application peut être étendue à l'analyse des discours que les enseignants tiennent sur leurs pratiques.

La construction des mondes d'éducation repose sur deux dimensions que Chatel (1997) estime fondamentales (cf. Tableau 1). La première se rapporte à l'identité que l'enseignant attribue à l'apprenant: identité individuelle si l'enseignant tient compte des particularités de chaque élève et collective ${ }^{2}$ si l'élève est considéré comme fondu dans le groupe-classe. La deuxième dimension porte sur la conception que l'enseignant a du savoir: savoir-substantif si l'enseignant considère le savoir comme des connaissances déposées dans les livres ou dans les objets, voire dans les événements ou anecdotes partagés en classe, et savoir-verbe s'il est considéré comme issu d'une réappropriation ou reconstruction par l'élève des connaissances des autres ou de celles déposées dans les livres ou dans les choses. 
Ainsi, les mondes d'éducation sont autant de scénarios possibles - mais non nécessaires - de l'action éducative. Ils fonctionnent chacun selon une logique propre et présentent une cohérence interne.

Tableau 1: Les mondes d'éducation (selon Chatel, 1997, p. 24, modifié)

\begin{tabular}{|c|c|c|}
\hline & $\begin{array}{c}\text { Savoir-verbe } \\
\text { Les savoirs sont considérés } \\
\text { comme une réappropriation des } \\
\text { connaissances d'autrui et de } \\
\text { celles qui sont inscrites dans les } \\
\text { livres ou dans les choses; activité } \\
\text { qui se nourrit de l'expérience }\end{array}$ & $\begin{array}{c}\text { Savoir-substantif } \\
\text { Les savoirs sont considérés } \\
\text { comme des connaissances ob- } \\
\text { jectives entreposées dans les } \\
\text { choses et dans les livres, com- } \\
\text { plètement descriptibles par les } \\
\text { mots et les règles }\end{array}$ \\
\hline $\begin{array}{l}\text { Identité individuelle } \\
\text { Les élèves sont considérés dans } \\
\text { leur individualité, avec leurs } \\
\text { particularités, avec leurs diffi- } \\
\text { cultés spécifiques, etc. }\end{array}$ & $\begin{array}{c}\text { Monde } \\
\text { didactique }\end{array}$ & $\begin{array}{c}\text { Monde } \\
\text { pédagogique }\end{array}$ \\
\hline $\begin{array}{c}\text { Identité collective } \\
\text { L'enseignant s'adresse avant tout } \\
\text { au collectif; l'élève est considéré } \\
\text { comme fondu dans le groupe- } \\
\text { classe. }\end{array}$ & $\begin{array}{l}\text { Monde } \\
\text { magistral }\end{array}$ & $\begin{array}{l}\text { Monde } \\
\text { scolaire }\end{array}$ \\
\hline
\end{tabular}

Dans le monde scolaire, on attend de tous les élèves qu'ils apprennent les règles ou le texte du savoir «exact» ${ }^{3}$, fidèle à la vulgate. Les principes d'évaluation sont relativement simples puisqu'on peut facilement dire ce qui est juste ou faux par référence au savoir «exact». L'enseignant maîtrise les dispositifs et les méthodes; les élèves écoutent, s'exercent, répètent, apprennent. Dans ce monde, l'enseignant pense qu'il y a synchronie entre enseignement et apprentissage.

Dans le monde pédagogique, l'enseignant conçoit aussi le savoir comme savoirsubstantif mais il prend en compte les particularités de chaque élève. L'évaluation repose sur les mêmes principes que dans le monde scolaire mais peut s'adapter aux caractéristiques des élèves (différenciation). Les élèves s'expriment durant l'enseignement et leur avis est réellement pris en compte; l'enseignant est un médiateur qui met son savoir à disposition et qui est prêt à aider chacun en particulier. Au niveau du temps didactique, on considère, comme dans le monde scolaire, qu'il suffit d'enseigner pour que les élèves apprennent.

Dans le monde magistral, les enseignants se représentent le savoir comme résultant d'une activité de l'apprenant. Dans une sorte de spectacle de la science en train de se construire, l'enseignant donne à voir sa relation personnelle aux connaissances et méthodes de sa discipline. Les élèves apprennent en l'imitant, ce qui nécessite un travail d'inférence important. Pour l'évaluation, il sera demandé à l'élève de problématiser une situation en faisant appel aux bonnes procédures, aux arguments cohérents; l'enseignant jugera seul de sa qualité. Dans cette perspective, le temps d'enseignement ne fait quamorcer les apprentissages qui se feront dans la durée. 
Dans le monde didactique, l'élève travaille à produire ses propres connaissances sur l'objet étudié; le maître est à l'écoute de ses élèves et invente les situations formatrices auxquelles confronter les élèves. L'évaluation sera une réalisation singulière de l'élève portant sur l'activité de savoir attendue. On considère que le temps d'apprentissage sera celui de l'appropriation par l'élève de cette activité de savoir.

Encore deux remarques à propos de ce modèle d'analyse. Au sujet de l'appellation de chaque catégorie, l'auteur précise: "ces mondes sont alternatifs, nous n'établissons pas d'ordre entre eux. Nous ne pensons pas l'un a priori meilleur que l'autre, ils sont le moyen pour nous d'analyser les situations effectives» (Chatel, 1997, p. 25). Pour ce qui est de l'utilisation de ces catégories dans l'analyse, on considère que l'action qui se déroule en classe ne se cantonne pas à un seul monde mais peut passer d'un monde à l'autre, en fonction du jeu des acteurs.

\section{Hypothèses}

Dans la géographie scolaire française, le glissement du modèle «républicain» vers le modèle «boîte à outils» a ouvert la porte à une grande variété de contenus, diversité que nous postulons retrouver en Valais. Nous faisons cependant l'hypothèse que cette diversité n'est qu'apparente et que les pratiques présentent des convergences sur le fond, sur les dispositifs d'enseignement, sur les démarches et exercices proposés aux élèves, sur l'évaluation et sur des contenus relevant de la vulgate; toutes ces convergences attesteraient d'habitudes disciplinaires bien établies.

\section{Dispositif de recherche et échantillonnage}

Vu que l'éducation helvétique s'organise au niveau cantonal, il nous est apparu nécessaire de nous limiter à un seul canton, en l'occurrence le Valais (partie francophone). Le degré d'enseignement a volontairement été restreint aux $5 \mathrm{e}$ et $6 \mathrm{e}$ primaires, de manière à garantir une certaine unité de l'analyse. Pour contacter les six enseignants interviewés, nous avons procédé de proche en proche, par réseau de connaissances. Sans prétendre à une quelconque représentativité des résultats, nous avons cependant cherché à diversifier les profils des personnes afin d'obtenir des discours aussi variés que possible. Les critères de diversification portaient sur l'âge, la formation, le sexe, le lieu d'enseignement (plaine/montagne, ville/village, Valais central/Bas-Valais).

Pour cette recherche prospective ${ }^{4}$, nous avons procédé à des entretiens semidirectifs (Ruquoy, 1995). Deux rondes d'entretiens ont été menées (mars-avril et fin juin 2001). D'une durée oscillant entre $1 \mathrm{~h}$ et $1 \mathrm{~h} 30$, le premier entretien avait pour but d'amener les six personnes à s'exprimer de manière assez libre sur leur enseignement en géographie. Pour le conduire, nous nous étions fixé un certain nombre de thèmes sur lesquels nous voulions les entendre tôt ou tard (formation 
et parcours professionnel, sujets abordés en géographie, démarches d'enseignement, documents utilisés, découpage horaire, lien avec d'autres branches, travail à domicile, relation avec les parents, plaisir à enseigner et plaisir des élèves en géographie, avis sur la société actuelle et valeurs personnelles, finalités de la géographie, réformes vécues, géographie connue en tant qu'enfant). Nettement plus court, le second entretien était destiné à obtenir des compléments d'information ciblés et a permis de récolter les copies des évaluations réalisées durant l'année ainsi que trois à quatre cahiers ou documents d'élève par classe. En tant que traces de ce que les enseignants jugent bon que les enfants retiennent, nous avons pensé que ces pièces constitueraient de précieux compléments à leurs discours.

En vue de l'analyse, le premier entretien a été complètement retranscrit alors que le second, complémentaire, a fait l'objet d'une retranscription uniquement lorsque son contenu s'avérait utile à l'analyse. Une étape de codage a été nécessaire pour étiqueter le contenu des discours; il s'est agi d'identifier les parties liées aux différents thèmes que nous nous étions fixé pour l'entretien semi-directif. De leur côté, les cahiers d'élèves et les évaluations ont été soumis à une analyse de contenu (présence/absence des différents sujets abordés en entretien).

\section{Analyses et résultats}

Les données récoltées (discours des enseignants, évaluations et cahiers d'élèves) sont considérées comme des traces de l'action que nous cherchons à catégoriser à l'aide des mondes d'éducation (Chatel, 1997). L'enjeu de l'analyse est de percevoir dans quel monde se situe l'action, et si l'action change de monde, quand et comment cela se réalise ainsi que par qui et pour quelle raison. Ce sont principalement les données codées sous le thème «démarche d'enseignement» qui ont été prises en compte dans cette analyse.

\section{La Logique d'action bipolaire}

Une première logique d'action se dégage nettement de trois entretiens. Elle est avant tout liée au traitement de la nomenclature. Ici, l'action se positionne en tension entre le monde pédagogique et le monde scolaire selon un mouvement précis qu'un enseignant nous fait percevoir:

[...] ce qu'ils vont réellement étudier, ici, c'est plus la nomenclature. [...] Là c'est eux qui recherchent, sur la carte de la Suisse. [...] ça prend beaucoup de temps quand même, plusieurs leçons, quand on a vu les chaînes et les cours d'eau, les montagnes et les cols; ça prend du temps surtout parce que les élèves les recherchent eux-mêmes. Et puis au tableau on note ce qui est validé: voilà, c'est juste, on a trouvé, et puis après ils copient sur des feuilles, puis ensuite ils étudient et puis ils font un test $[. .$. 
Dans cette logique, l'action débute généralement dans le monde pédagogique par toute sorte d'activités pour "découvrir» la nomenclature, savoir-substantif par excellence. En se référant à diverses cartes ou documents, l'élève est généralement appelé à établir une liste de noms ou à compléter une carte muette. Le but est que chacun, à sa manière, se familiarise avec ces mots ou localisations en les manipulant, en les positionnant sur carte, en les organisant par rapport à ses repères personnels, en construisant des liens entre eux, etc.

Après un temps plus ou moins long consacré à ce jeu d'identification et d'appropriation où le tâtonnement individuel est de mise, l'action bascule dans le monde scolaire où il s'agit de mémoriser la nomenclature et de procéder à l'évaluation. Finies les particularités individuelles, balayées les références au vécu de chacun, puisque dans ce monde, seuls le mot ou la localisation corrects comptent, et cela pour tous les élèves de la même manière. La transition d'un monde à l'autre se déroule par une étape de validation ou d'institutionnalisation, moment où l'enseignant, par exemple, s'assure que les enfants ont relevé correctement les mots à apprendre. Ce dernier détail montre qu'une erreur dans le nom ou la localisation d'un objet est considérée différemment selon que l'on est dans le monde scolaire ou dans le monde pédagogique.

Simple et rodée, cette logique de travail est à tel point intériorisée par les enseignants et les élèves qu'il est difficile d'en sortir. En effet, dans le cadre d'une évaluation et pensant faire preuve de générosité, un enseignant décide au dernier moment d'autoriser le recours à la carte et l'annonce au début du travail des élèves. Ce faisant, il donne la possibilité de déplacer l'action évaluative dans un autre monde (mondes des savoirs-verbes). Il constate cependant avec surprise que les élèves ont très peu utilisé leur carte. Habitués à la logique bipolaire, ceuxci semblent préférer «jouer» leur évaluation dans le monde scolaire dont ils mầtrisent les tenants et aboutissants.

\section{La Logique d'action triangulaire}

Plus globale et très fréquente (tous les enseignants la mettent en œuvre), la logique d'action triangulaire est utilisée pour traiter de thèmes tels que relief, climat, glacier, végétation, ville, etc. soit pour eux-mêmes, soit au travers de l'étude de différents paysages. Sur ces objets plus complexes que la nomenclature, l'action triangule. Elle démarre dans le monde pédagogique où l'enseignant va "chercher» les élèves en faisant un important travail d'explicitation du savoir, très proche d'eux. Ce travail d'éclaircissement l'amène à faire des raisonnements avec ses élèves et l'action se déplace alors temporairement en direction du monde magistral ou parfois aussi didactique. Finalement, après s'être rapproché des élèves, l'enseignant leur demande de "coller» aux savoirs qu'il veut leur faire acquérir et l'action passe ainsi dans le monde scolaire pour l'évaluation. Comme dans la logique précédente, ce passage se fait par un jeu d'institutionnalisation rôdé qui peut prendre différentes formes et dont le produit est un savoir-substantif à mémoriser (vocabulaire spécifique, caractéristiques, raisons ou schémas explicatifs, etc.). 


\section{Une particularité: la phase d'explicitation}

Cette logique se distingue de la logique bipolaire par la phase d'explicitation qui y tient un rôle primordial. Ce travail se fait en commun, par un jeu de questionréponse et d'explication, comme l'atteste cet enseignant qui travaille sur le paysage du Bas-Valais.

[...] on essaie de placer les choses et puis chacun apporte un peu ses expériences [...]. Après on est parti avec le livre et on a fait les lectures. On a essayé de comprendre ce qui se passait; je leur ai amené des explications, parce que là, au niveau du relief, c'était assez délicat [...]. On a travaillé en commun oralement; les élèves ont aussi dî discuter entre eux et puis après apporter des choses [...]. Et puis on lit ensemble; en fait [...] ils ne les comprennent pas vraiment ces textes quand ils les $l i-$ sent; il n'y a que des petites choses quils saisissent alors après il faut replacer les choses. Et puis au début on insiste aussi sur la carte, où c'est placé Monthey, oralement qu'est-ce qu'on peut dire [...]

On voit que dans cette phase, l'enseignant va tout faire pour mettre le savoir à portée des élèves afin qu'ils puissent mieux se l'approprier: il le reformule, le met en relation avec ce qui est connu ou supposé connu, l'illustre par des documents de toutes sortes, l'exemplifie par le vécu, le modélise, etc. Mobilisant la relation personnelle que les élèves entretiennent avec l'objet étudié et s'arrêtant sur leurs difficultés et leurs questions particulières et désirant que chacun puisse comprendre, l'enseignant donne à l'apprenant une identité individuelle. Pour ce qui est des savoirs en jeu, le travail de la phase d'explicitation porte le plus souvent sur des savoirs-substantifs ou, en d'autres termes, sur les connaissances qu'il faudra mémoriser. Elle se positionne donc avant tout dans le monde pédagogique, caractérisé par une atmosphère sympathique: les élèves se sentent concernés, ils participent et sont intéressés.

Cependant, selon la tournure que prend l'interaction, l'action de la phase d'explicitation peut aussi se déplacer dans les mondes des savoirs-verbes. En effet, tout en cherchant à leur faire comprendre des savoirs-substantifs, l'enseignant donne à voir aux élèves comment travailler sur les savoirs, comment soumettre textes, schémas et graphiques au questionnement, comment se montrer critique face à ceux-ci, comment résumer ou faire une synthèse, comment faire des comparaisons ou des liens entre les choses, comment problématiser une situation et en discuter, etc. Montrant ainsi la face cachée des connaissances qu'il donne à apprendre aux élèves, l'enseignant déplace alors l'action dans le monde magistral. Dans d'autres circonstances, il met les élèves au travail sur une activité individuelle qui leur demande d'observer, de comparer, d'établir des liens et d'émettre des hypothèses; l'action se déroule alors dans le monde didactique. Un troisième cas de figure apparaît; il s'agit d'un monde intermédiaire, que l'on pourrait appeler magistro-didactique, où l'enseignant s'appuie sur quelques élèves et leur demande de «faire le maître», c'est à dire d'exprimer à sa place un certain nombre d'idées ou de raisonnements plus complexes. Dans tous ces mondes, l'élève apprend par imitation progressive ${ }^{5}$, de façon différée, et son métier d'élève est assez 
difficile. Cependant, la plupart des enseignants disent vouloir favoriser le développement de ces savoir-faire ou d'un certain raisonnement géographique ${ }^{6}$.

L'analyse des entretiens nous permet de conclure que la phase d'explicitation, cœur de la logique triangulaire, se décline sur les deux versants du savoir (savoirsubstantif et savoir-verbe). Cette double dimension est peut-être la raison qui explique sa popularité; à entendre la valeur que les enseignants lui conferent, cette phase semble même constituer tout l'art de la profession, la suite n'étant finalement qu'un processus de certification. Plusieurs enseignants nous ont d'ailleurs fait part d'un réel plaisir à mener ce travail d'explicitation, à accompagner et soutenir les enfants dans leur découverte et compréhension du savoir, à se questionner et à raisonner ensemble sur le monde.

S'il ne fait aucun doute, selon les discours des enseignants, que les savoirsverbes sont travaillés dans la phase d'explicitation, force est de constater qu'ils n'apparaissent ni dans les documents d'élève, ni dans les évaluations. Et les quelques exceptions observées confirment la règle, puisque les rares raisonnements qu'on y rencontre ont tellement été réifiés et figés dans le processus d'institutionnalisation qu'ils en deviennent des savoirs-substantifs. Prenons l'exemple d'une des questions d'évaluation analysées: «quelles sont les conditions pour avoir un glacier?». Apparemment, elle fait appel à la réflexion, mais on s'aperçoit bientôt, par les deux lignes qui suivent la question, que la réponse attendue se limite aux deux éléments qui figurent dans le cahier des élèves, à savoir «de la neige» et «du froid». Dans la logique triangulaire, les savoirs-verbes ne passent donc pas le seuil de l'institutionnalisation; l'évaluation se joue toujours dans le monde scolaire.

\section{Effet des élèves sur le déroulement de la logique triangulaire}

Dans cette logique comme dans la logique bipolaire, les mouvements sont principalement imprimés par l'enseignant, qui garde la main mise sur l'action dans une sorte de démarche frontale améliorée et qui a des intentions claires: ramener progressivement l'action dans le monde scolaire. Cependant, les élèves peuvent aussi influencer le déroulement de la logique ou la contrecarrer.

Dans la phase d'explicitation, il est attendu de l'élève qu'il cherche à comprendre, interpelle le maître si quelque chose ne lui paraît pas clair, donne son idée ou son avis, etc. Mais il peut arriver que les élèves refusent d'entrer dans ce rôle qui relève du monde pédagogique et veulent placer directement l'action dans le monde scolaire, sachant pertinemment que c'est dans celui-ci qu'il leur faudra rendre compte du savoir. La logique risque alors le blocage:

Cette année, ça marche bien parce que c'est une classe très active et qui participe.

Il y a des années où c'est rude, où des moments teen as marre de procéder comme ça; tu aurais envie de donner la matière comme ça en disant "voilà comment ça se passe», parce que tu poses des questions et c'est toi qui réponds.

La logique triangulaire peut aussi bloquer - ou du moins ne pas porter ses fruits - si les élèves ne parviennent pas à suivre les déplacements de l'action entre les 
mondes ou s'ils considèrent chacun d'eux de manière indépendante. C'est ce qui se passe pour certains élèves qui présentent des difficultés de mémorisation et qui, par conséquent, ont de mauvais résultats. En fait, on constate que lorsque l'enseignant procède à la phase d'explicitation et qu'il place l'action dans le monde pédagogique, il suspend volontairement la pression du monde scolaire et ses impératifs d'évaluation - ce qui contribue à la mise en place d'une atmosphère détendue - mais il conserve un certain nombre d'attentes fortes sur les savoirs et leur assimilation. Ces attentes échappent cependant à quelques élèves pour qui l'action conviviale qui se déroule dans le monde pédagogique ne représente qu'un jeu ou une causerie en soi. Lorsqu'ils préparent l'évaluation, ces élèves repartent à zéro; leur travail de mémorisation est donc considérablement plus complexe que croient les enseignants. Ceux-ci, pensant qu'il suffit souvent de voir pour comprendre et d'écouter pour retenir, le considèrent comme relativement facile car en continuité avec la phase d'explicitation.

\section{La Logique didactique}

Une autre régularité de pratique a pu être mise en évidence grâce aux entretiens. Cette logique d'action que quatre enseignants tentent de mettre en œuvre porte avant tout sur l'analyse du paysage telle que développée dans la méthodologie officielle (Mudry, 1989, 1993). Elle doit permettre à l'élève d'entreprendre une démarche d'analyse de paysage en allant rechercher les informations dans des documents de toutes sortes (cartes, photos, schémas, graphiques, etc.). Si cette recherche repose essentiellement sur les épaules de l'élève, le rôle de l'enseignant est de le mettre au travail et de l'accompagner dans sa démarche.

Du point de vue de son déroulement, cette logique s'en tient presque exclusivement au monde didactique. Elle intervient toujours à la suite d'un travail d'analyse de paysage réalisé à l'aide de la logique triangulaire. Ainsi, dans la logique didactique, les élèves sont invités à réaliser, individuellement et sur une nouvelle région, la démarche d'analyse qui avait été faite collectivement auparavant. Une mise en commun est ensuite nécessaire pour partager et confronter les analyses personnelles. Pour l'évaluation, l'élève doit entreprendre une démarche d'analyse sur un paysage qui n'a encore jamais été travaillé. Dans ce cas, ce ne sont pas les connaissances en tant que telles qui intéressent l'enseignant, mais la capacité des élèves à chercher des informations et surtout à raisonner sur celles-ci ${ }^{7}$. Prise comme telle, cette logique d'action qui se cantonne résolument au monde didactique présente une certaine cohérence interne qui en assure le bon fonctionnement et donne satisfaction à l'enseignant.

Mais les entretiens montrent que cette logique présente plusieurs difficultés majeures. Tout d'abord, le métier d'élève est particulièrement ardu vu qu'il s'agit de mettre en œuvre des savoirs-verbes rarement travaillés pour eux-mêmes. La manière dont un enseignant parle de certains élèves qui mendient (et obtiennent parfois) les réponses montre que ceux-ci préferent porter l'action dans le monde pédagogique, plus convivial, où il existe une «bonne» réponse que le maître finit 
toujours par donner. Ensuite, le monde didactique, basé sur le travail de l'élève qui avance à son rythme, présente le risque que l'activité ne puisse être achevée. Un enseignant illustre magnifiquement ce problème en montrant comment, de remédiation en remédiation, certains ne terminent jamais leur travail. La mâ̂trise des contenus (notamment en géographie humaine) est également une difficulté évoquée par certains enseignants qui peuvent se sentir désarçonnés par les questions que les élèves posent parfois dans ce genre de démarches ouvertes. Finalement, et c'est peut-être la difficulté majeure pour l'enseignant, le monde didactique ne se prête pas à l'évaluation sous sa forme habituelle; ce qui s'y développe ne se laisse pas «écrire» comme des connaissances déclaratives; la validation des acquis doit obligatoirement être pensée différemment.

Toutes ces raisons font qu'il est difficile, pour un enseignant, de maintenir l'action dans le monde didactique; cette dernière a souvent tendance à se déplacer dans un autre monde. Ce genre de déplacement est précisément ce qui se passe pour trois des quatre enseignants: ils valident le travail effectué dans le monde didactique en utilisant les critères d'appréciation du monde scolaire. En fait, cela contribue à court-circuiter tout le travail effectué en amont car les élèves comprennent assez vite que les efforts et le travail qu'ils fournissent dans le monde didactique ne seront pas vraiment évalués ni valorisés. Ce genre de glissement apparaît chez les enseignants qui considèrent l'activité sur le paysage comme une façon originale de «découvrir» les connaissances à mémoriser. En vue de leur évaluation dans le monde scolaire, ils souhaitent que les résultats de la démarche soient soignés, corrects et semblables pour tous. Or, dans le monde didactique, les productions des élèves sont souvent très variables, tant en qualité qu'en quantité ${ }^{\prime}$, si bien que les enseignants concernés par ce glissement se déclarent peu satisfaits de la démarche d'analyse de paysage prônée par la méthodologie officielle.

\section{Conclusion}

Sans autoriser de généralisation, l'utilisation des mondes d'éducation (Chatel, 1997) dans l'analyse des six discours, des évaluations et des cahiers d'élève nous a permis de mettre de l'intelligibilité dans des pratiques scolaires à première vue disparates. Nous avons constaté que les actions décrites passent d'un monde à l'autre en s'enchaînant selon des logiques construites, cohérentes du point de vue de l'enseignant, et récurrentes dans les entretiens. Les trois logiques d'action distinguées mettent ainsi en évidence certaines convergences de fond que nous détaillons dans cette conclusion, avant d'aborder les aspects méthodologiques et quelques considérations d'ordre pratique.

Apports de la recherche à la connaissance des pratiques Au niveau des contenus, passé une certaine diversité qui a pu être mise en évi- 
dence dans un autre volet de la recherche (Fierz, 2002, chapitre 2) ${ }^{9}$, un certain nombre de convergences majeures apparaissent. La nomenclature est omniprésente, de même que des thèmes géographiques typiques (relief, climat, végétation, éléments humains) qui sont abordés soit séparément et de manière approfondie, soit l'un après l'autre, brièvement, au travers d'une étude de paysage dont ils constituent la grille de lecture. Selon que les découpages considérés sont politiques ou naturels, les espaces étudiés sont le canton du Valais ou les Alpes en 5e primaire, la Suisse respectivement le Jura et le Moyen-Pays en 6e primaire.

Les convergences sont encore plus manifestes sur les questions de fond. Que l'on travaille sur la nomenclature, sur des thèmes ou sur des paysages, la progression est sensiblement la même (circulation entre les mondes d'éducation selon une logique que nous avons appelée bipolaire ou triangulaire). Le savoir est tout d'abord explicité - l'enseignant fait ressortir les idées des élèves, les oppose entre elles ou à des documents, amène des modèles explicatifs et des informations, convoque le vécu, propose quelques exercices individuels ou par groupe - puis il est institutionnalisé, selon des modalités variées, afin que l'élève sache ce qu'il doit apprendre et ce sur quoi il va être évalué. Cette progression de caractère inductif(elle raccroche le savoir nouveau à ce que l'élève est censé déjà connaître ou ce qui est jugé à sa portée) est en grande partie liée à la conception que ce qui est «proche» des élèves, ce qui est concret ou vécu, permet une meilleure compréhension.

Les savoirs-substantifs sont majoritaires et l'on attend le plus souvent des enfants qu'ils puissent nommer, citer, localiser, énumérer et décrire des objets ou reproduire une explication. Les savoirs-verbes ne sont pas oubliés puisque les enseignants les jugent primordiaux et que des éléments de méthode et de raisonnement sont souvent travaillés dans la phase d'explicitation du savoir (des contradictions sont posées par l'enseignant, des réflexions, des hypothèses, des comparaisons sont faites par les élèves). Cependant, ces savoirs-verbes ne sont pas évalués, ce qui diminue grandement l'attention qu'on leur porte.

Ces constances bien établies montrent la persistance de certaines formes scolaires et attestent de l'influence que l'impératif d'évaluation a sur les pratiques. On a pu montrer que l'évaluation se joue presque exclusivement dans le monde scolaire. Une des raisons majeures réside dans les conceptions des enseignants; en effet, pour eux, l'évaluation devrait être «juste» - d'où l'importance d'évaluer tous les élèves sur les mêmes savoirs - et «objective» - d'où les convergences autour des savoirs-substantifs que l'on peut déclarer justes ou faux sans équivoque. Par conséquence, en amont de l'évaluation, le travail de classe se déplace tôt ou tard dans le monde scolaire, le seul monde considéré comme légitime.

Mais ces constances ont leurs exceptions. A quelques moments de l'année, certains enseignants adoptent une logique d'action complètement différente, mettant l'enfant au travail dans la perspective de construire des savoirs-verbes (logique didactique). Cependant, plusieurs peinent à maintenir l'action ancrée sur les savoirs-verbes jusqu'à l'évaluation; de multiples raisons (comportement des 
élèves, conceptions de la géographie, objectifs non clarifiés, traditions d'évaluation, etc.) engendrent des glissements vers les savoirs-substantifs. Les difficultés liées à cette logique de travail viennent conforter les habitudes scolaires mises en évidence, en montrant combien elles sont prégnantes et combien il est difficile d'inventer et de maîtriser d'autres formes scolaires.

Ces résultats sont assez proches de la vaste enquête qu'Audigier (1999) a menée en France auprès des enseignants des trois dernières années du primaire ${ }^{10}$ et qui a combiné à la fois dispositif d'entretien (au nombre de 46) et questionnaire à plus large représentativité (859 questionnaires). Les habitudes scolaires identifiées vont aussi dans le sens d'autres recherches qui ont étudié les pratiques scolaires françaises sur des terrains et selon des problématiques variés: Audigier \& Basuyau (1988); Audigier (1993); Audigier, Crémieux \& Mousseau (1996); Basuyau \& Guyon (1994); Crémieux, Jacob \& Mousseau (1994). Par rapport à toutes ces connaissances déjà construites sur les pratiques scolaires en géographie, le principal intérêt de notre recherche est d'avoir montré qu'au-delà des différences de systèmes éducatifs (France, Suisse romande), on retrouve les mêmes convergences. Faut-il y voir l'influence de l'aire culturelle francophone ou l'empreinte de la forme scolaire? Une recherche dans les cantons suisses alémaniques ou dans d'autres aires linguistiques permettrait d'en débattre ${ }^{11}$.

\section{Apports méthodologiques}

D'un point de vue méthodologique, la combinaison des traces (discours construits par entretiens semi-directifs, évaluations effectuées durant l'année et cahiers d'élève) s'est avérée pertinente pour explorer les pratiques et mettre en évidence les habitudes disciplinaires. Même si les résultats ne peuvent être considérés comme représentatifs vu la taille de l'échantillon, les convergences marquées auxquelles nous sommes parvenu, et ce malgré les critères de diversification que nous avons adoptés lors de l'échantillonnage (âge et expérience, lieu d'enseignement, sexe), donnent une certaine crédibilité à ces régularités. Mais l'innovation méthodologique de notre recherche est d'avoir montré que les mondes d'éducation construits par Chatel (1997) pour rendre compte de l'action éducative en train de se faire (approche par observation) peuvent aussi être appliqués à l'analyse de discours sur l'action (approche par entretien) moyennant la précaution de travailler simultanément sur des traces complémentaires tels que cahiers d'élèves et évaluations. A notre connaissance, cela n'avait encore jamais été entrepris; en effet, après avoir présenté son modèle, Chatel parle de son application à l'analyse de résultats de l'action éducative, plus précisément des évaluations et des notes, mais ne signale aucune tentative d'analyse de discours.

Cette recherche a également montré toute la pertinence de l'approche didactique, qui, grâce à des outils d'analyse spécifiques, permet l'étude du système didactique dans sa globalité, sans dévier ni se focaliser sur l'une ou l'autre de ses composantes. Développés en didactique des sciences sociales, les mondes d'éducation constituent un de ces outils intégrateurs car il porte sur l'analyse de l'action 
qui se noue entre enseignant et élèves dans la négociation du savoir, négociation où chaque acteur a des intentions, prend des décisions et agit pour modifier le cours de l'action éducative. Un autre concept d'analyse présente le même intérêt; il s'agit du contrat didactique développé par Brousseau (1998) en didactique des maths. Certains didacticiens, comme par exemple Schubauer-Leoni, Leutenegger \& Mercier (1999), étudient l'évolution du contrat didactique en se centrant sur l'analyse de la tâche et sa négociation en classe. Un peu comme nous avons analysé le déplacement de l'action d'un monde à l'autre, ces chercheurs étudient les enjeux qui se nouent et se dénouent implicitement autour de la tâche et qui mettent en jeu l'enseignant - qui va proposer des déplacements de la tâche - les élèves - qui vont essayer de percevoir ce que l'enseignant attend d'eux pour l'honorer - et les savoirs - qui sont censés être construits au travers de la tâche et dont enseignants comme élèves se font une représentation particulière. Nous pensons que d'intéressantes comparaisons conceptuelles seraient à faire entre ces deux cadres d'analyse didactique. Il serait aussi utile d'étudier leur adéquation avec une discipline plutôt qu'avec une autre; en effet, peut-on étudier avec les mêmes outils l'enseignement des mathématiques, où il est important de «savoir résoudre», et celui de la géographie reposant en grande partie, comme nous l'avons vu, sur «savoir que [...]»?

Toujours sur le plan méthodologique, cette recherche a également permis de tester la portée du modèle d'analyse construit par Chatel (1997). Son pari (p. 23) était de proposer un modèle sobre qui puisse tout de même cerner l'essentiel des phénomènes d'enseignement/apprentissage. Dans notre recherche, la pertinence de ce modèle a pu être évaluée par un autre volet (Fierz, 2002, chap. 4 et 6, 7, 8). Indépendamment de l'utilisation du modèle de Chatel, nous avons étudié les choix des enseignants et les arguments qu'ils utilisent pour les justifier. Il s'avère que les justifications les plus fréquentes et qui influencent le plus fortement les pratiques sont leurs conceptions de l'apprentissage (importance conférée à l'intérêt supposé des élèves, à leur vécu, à ce qui est proche et concret; refus du complexe) et leurs conceptions épistémologiques (ce qu'est la géographie et ses objets, ce qu'il faut faire pour acquérir ce savoir). Or, les deux dimensions retenues par Chatel questionnent précisément ces points-là:

1. Conception du savoir: Le savoir est-il déposé dans les choses (dans ce cas, il suffit de regarder pour apprendre, d'où l'importance du concret et du vécu) ou implique-t-il une reconstruction par l'apprenant? Faire de la géographie, estce mémoriser des savoirs-substantifs ou faire construire des savoirs-verbes?

2. Conception de l'apprenant: Veut-on le même savoir pour tous ou accepte-t-on de les différencier? Tient-on compte des apports des élèves et de ce qu'ils savent déjà (différences interindividuelles)?

En plus des conceptions épistémologiques et de l'apprentissage, les mondes d'éducation intègrent également aussi les questions d'évaluation - dont nous avons vu l'importance ci-dessus - puisqu'à chacun d'eux correspond une forme d'évaluation particulière. Evidemment, même si notre recherche a montré la pertinence 
de ce modèle qui tient compte des conceptions des acteurs, il reste un outil parmi d'autres, avec ses catégories et son point de vue.

\section{Apports pratiques}

Cette recherche amène également quelques éléments utiles aux pratiques. Elle permet tout d'abord d'interroger les réformes scolaires. En effet, l'analyse de paysages - travaillée selon la logique didactique - constitue l'essentiel de la réforme entreprise dans l'enseignement de la géographie au cours des années 1980 en Valais. Or, une quinzaine d'années plus tard, force est de constater que cette approche est peu suivie ${ }^{12}$.

Les raisons sont bien évidemment multiples. Certaines ont pu être identifiées dans une autre partie de la recherche (Fierz, 2002, chap. 6). Constatant que même les jeunes enseignants interviewés dans cette recherche pratiquent peu la nouvelle approche alors qu'ils y ont été formés à l'Ecole normale, nous pensons que le poids des habitudes disciplinaires ainsi que l'impact des conceptions sur ce qu'est la géographie ont une importance majeure. En étudiant la véhémence des discours tenus par certains enseignants ayant vécu la réforme, on s'aperçoit aussi que certaines raisons sont étroitement liées à la manière dont s'est déroulé le changement, selon eux trop rapide et mené sans véritable accompagnement, ni soutien matériel.

Dans tous les cas, nous faisons l'hypothèse qu'un changement aussi radical (développer les savoirs-verbes de l'élève par un travail autonome), devrait nécessairement passer par un travail de fond sur les conceptions que les enseignants acteurs incontournables de la réforme - ont de la géographie, de ses buts et de ses objets; Davaud \& Varcher (1990) estiment par ailleurs que même les réflexions qui ont conduit l'institution à faire le choix d'un nouveau projet éducatif devraient impérativement être partagées avec les enseignants. Cette recherche nous a également convaincu que dans un tel revirement, l'institution devrait développer des moyens pour évaluer ce type de savoir et inviter tous les acteurs de l'enseignement (parents compris) à entrer dans d'autres formes d'évaluation, à "accepter des progrès individualisés, des constructions inachevées, des savoirs inégaux» (Gérin-Grataloup, Solonel \& Tutiaux-Guillon, 1994, p. 35).

Le deuxième apport pratique que nous identifions concerne la formation des futurs enseignants. Afin de les aider à prendre une distance critique par rapport aux coutumes didactiques, nous pensons qu'une grille d'analyse telle que les mondes d'éducation (Chatel, 1997) pourrait s'avérer fort utile.

Dans les cours de didactique d'une part, cet outil relativement simple à comprendre et à manipuler permettrait de clarifier deux dimensions de première importance pour les apprentissages que sont les savoirs et la place de l'apprenant. Ces catégories permettraient de questionner, par exemple, les conceptions largement répandues autour du savoir, considéré comme déposé dans les choses et directement appréhendable (il suffirait de voir pour comprendre, d'entendre pour savoir), conceptions qui mériteraient d'être revisitées. 
Sur le terrain d'autre part, cet outil d'analyse permettrait à l'enseignant-stagiaire d'être introduit à la complexité des phénomènes d'enseignement/apprentissage, d'y mettre de l'ordre et de réfléchir à la manière de travailler dans cette complexité. Selon que l'on considère l'apprentissage, les conditions de mises en œuvre, le pouvoir motivationnel, le temps nécessaire, l'évaluation et la communication avec les parents, etc., chacun de ces mondes présente à la fois des avantages et des inconvénients. Les mondes d'éducation ne sont donc pas des modèles de références à confronter; ils sont des modes d'action fréquemment utilisés en classe - de manière consciente ou non - et dont il faut percevoir les avantages et inconvénients respectifs, comprendre le métier d'élève auquel ils renvoient, comprendre le rôle de l'enseignant qu'ils requièrent pour pouvoir fonctionner. Esquissons brièvement les atouts de chaque monde tout en se rappelant que les avantages mis en avant pour un monde constituent souvent ce qui fait défaut aux autres:

- Le monde didactique présente des avantages du point de vue des apprentissages car il offre un espace où tous les élèves peuvent construire individuellement des «savoir raisonner» en même temps que des «savoir que [...]».

- Le monde pédagogique a l'avantage d'être très motivant et toujours convivial. Cette mobilisation des élèves est un levier important pour l'apprentissage.

- Le monde scolaire assure à l'enseignant un ancrage sans faille dans le système scolaire car il constitue le monde le plus fortement légitimé par la société et les parents ${ }^{13}$.

- Le monde magistral permet de travailler sur des savoirs-verbes de manière économique et selon des dispositifs plus légers que dans le monde didactique.

\section{Quelle géographie enseigner?}

Les deux apports pratiques que nous venons de présenter portent sur le même enjeu, à savoir les conceptions que les enseignants ont de la géographie. Comme notre analyse l'a montré, une certaine coutume didactique (résistant aux réformes!) veut qu'elle soit considérée comme une discipline qui autorise les discussions sur la marche du monde mais qui, in fine, se limite à faire acquérir des connaissances sur ce monde.

En ce début de 21e siècle, alors que nous sommes submergés d'informations, que l'accès aux connaissances est rendue si facile, que la mondialisation brouille complètement nos échelles de référence, que la complexité des phénomènes engendre un relativisme discutable et une certaine démission, cette habitude disciplinaire nous paraît plus que jamais à questionner. Les finalités de la géographie ne devraient-elles pas être repensées de manière plus large en intégrant une dimension critique (construire un appareil critique pour raisonner sur le monde) et civique (se comprendre comme acteur de ce monde et construire une attitude plus responsable à l'égard des sociétés humaines, de leurs échanges, de leur cadre de vie). L'enseignement d'une telle géographie impliquerait nécessairement une conception plus constructiviste du savoir, ce qui reviendrait, en terme de mondes 
d'éducation, à travailler davantage dans les mondes didactique (en acceptant que cela prennent du temps et que les progrès soient différenciés) et magistral (en se méfiant du conditionnement - imitation du maître - qu'il induit).

\section{Notes}

1 Par mesure de commodité rédactionnelle, nous écrirons enseignant au masculin tout en priant le lecteur de lire enseignant(e).

2 Chatel parle d'identité générique; identité collective nous paraît cependant plus approprié.

3 Les guillemets sont de Chatel.

4 Aucune recherche n'a été menée sur l'enseignement de la géographie au primaire en Valais. En Suisse romande, seuls quelques rares auteurs se sont intéressés à la géographie scolaire, en privilégiant le niveau secondaire (Collet \& Hertig, 1995; Hertig, 1989).

5 Not (1987) rappelle que l'imitation n'est pas une simple reproduction par effet de miroir; elle implique tout à la fois prise de conscience, analyse de ses propres actes, comparaison, critique, ajustement et vision globale de la conduite à réaliser.

6 Laurin (1998, p. 18) définit le raisonnement géographique comme une «habileté de mémorisation, d'observation ou de description doublée de capacités de questionnement, de raisonnement, d'analyse, de synthèse, de jugement critique».

7 On ne peut pas exclure que l'élève procède à des transferts ou des inférences à partir de démarches d'analyse déjà effectuées; si cela était le cas, nous classerions aussi ces activités dans le monde didactique car, à notre sens, «transférer» ou «inférer» constituent des savoirsverbes qui participent à la reconstruction des savoirs.

8 Dans la logique didactique qui donne à chaque élève une occasion de construire une analyse et de se mettre en situation de raisonnement, les productions individuelles constituent une «documentation» de l'activité intellectuelle engagée par chacun en particulier; elles devraient donc être évaluées comme telle.

9 Il s'avère que les contenus sont relativement diversifiés, surtout à un certain niveau de détail. Cela est fortement lié aux opportunités de toutes sortes qui se présentent aux enseignants (documents, actualité, opportunités locales, etc.).

10 Les trois derniers degrés du primaire français correspondent aux degrés 3P (CE2), 4P $(\mathrm{CM} 1)$ et $5 \mathrm{P}(\mathrm{CM} 2)$; le degré $6 \mathrm{P}$ valaisan équivaut au début du secondaire français (6e) où l'enseignement n'est plus pris en charge par des généralistes.

11 Pour que le débat ne soit pas vidé de sa substance, il nous paraît important de dépasser le seul constat de similitude / différence pour se questionner sur ce qui justifie les habitudes scolaires en géographie. Sur ce point, notre recherche a montré que les pratiques étudiées sont liées d'une part aux représentations que des enseignants ont de la géographie (nature de la discipline, types de savoirs et de démarches qui lui sont liés) ainsi que de ce qui «marche» auprès des élèves (ce qui les intéresse, ce qui est concret ou en rapport avec leur vécu) et, d'autre part, sont liées à leur expérience (d'enseignant, d'étudiant ou d'écolier) ou à l'obligation d'évaluer les apprentissages. Ces éléments constituent à notre sens les principaux critères utilisés par les enseignants pour interpréter des programmes de géographie très larges et généraux.

12 Le concept de discipline scolaire qui considère que c'est le monde scolaire qui fabrique ses objets d'enseignement est donc tout à fait pertinent puisqu'on observe que l'approche par paysage voulue par la réforme s'est généralisée en tant que contenu (on aborde des paysages), mais que la façon de le travailler reste principalement cantonnée à la logique triangulaire.

13 Le sens commun veut en effet qu'à l'école, l'élève apprenne "sa" géographie, soit un certain nombre de connaissances semblables pour tous. 


\section{Bibliographie}

Audigier, F. (1993). Les représentations que les élèves ont de l'histoire et de la géographie. Thèse pour le doctorat de didactiques des disciplines, Université de Paris VII.

Audigier, F. (1995). Histoire et géographie: les savoirs scolaires en question entre les définitions officielles et les constructions des élèves. Spirale, Université de Lille, 8, 61-89.

Audigier, F. (1999). Les représentations de la géographie dans l'enseignement primaire en France. Cahier de Géographie du Québec, 43, (120), 395-412.

Audigier, F. \& Basuyau, C. (1988). Comment l'histoire et la géographie sont-elles enseignées? Exemple des classes de CM2 et 6e. Revue Française de Pédagogie, 85, 21-27.

Audigier, F., Crémieux, C. \& Mousseau, M.J. (1996). L'enseignement de l'histoire et de la géographie en troisième et en seconde. Paris: INRP.

Basuyau, C. \& Guyon, S. (1994). Consignes de travail en histoire-géographie: contraintes et libertés. Revue Française de Pédagogie, 106, 39-46.

Brousseau G. (1998). Théorie des situations didactiques. Textes rassemblés et préparés par Balacheff, N. \& al. Grenoble: La Pensée sauvage.

Chatel, E. (1997). Les mondes d'éducation, des catégories pour saisir la diversité de l'action éducative. In INRP (Ed.), Concepts, modèles et raisonnements. (pp. 19-33). Paris: INRP.

Chervel, A. (1988). L'histoire des disciplines scolaires: réflexions sur un domaine de recherche. Histoire de l'éducation, 38, 59-119.

Collet, G. \& Hertig, P. (1995). Du bon usage de l'image dans l'enseignement de la géographie. Geographica Helvetica, 95, (4), 138-140.

Crémieux, C., Jakob, P. \& Mousseau, M.-J. (1994). Regard didactique sur les productions scolaires en histoire-géographie. Revue Française de Pédagogie, 106, 47-54.

Davaud, C. \& Varcher, P. (1990). La géographie à l'école: entre objets de savoir, pratiques scolaires et démarches géographiques. Bildungsforschung und Bildungspraxis, 12, (3), 223-243.

Fierz, S. (2002). Enseignement de la géographie au primaire: quoi? pourquoi? Entretiens avec six enseignants valaisans de $5 e$ et Ge primaire. Mémoire de DEA en Sciences de l'éducation, Université de Genève.

Gérin-Grataloup, A.-M., Solonel, M. \& Tutiaux-Guillon, N. (1994). Situations-problèmes et situations scolaires en histoire-géographie. Revue Française de Pédagogie, 106, 25-37.

Hertig, P. (1989). Tendances de la géographie scolaire en Suisse romande. Quelques réflexions d'ordre didactique. Geographica Helvetica, 89, (1), 46-52.

INRP (1988). Savoirs enseignés - savoirs savants. Actes de la Rencontre nationale sur les didactiques de l'histoire de la géographie, des sciences sociales de 1988.

Laurin, S. (1998). Formation du citoyen et conscience territoriale. In J.-L. Klein \& S. Laurin (Ed.), L'éducation géographique. Québec: Presses de l'Université du Québec.

Lefort, I. (1992). La lettre et l'esprit. Paris: CNRS.

Mudry, A. (1989). Le paysage alpin. Méthodologie. A la découverte de la géographie. Sion: DIP Valais.

Mudry, A. (1993). Paysages du Jura et du Moyen-Pays. A la découverte de la géographie. Sion: DIP Valais.

Not, L. (1987). Enseigner et faire apprendre. Toulouse: Privat.

Ruquoy, D. (1995). Situation d'entretien et stratégie de l'interviewer. In L Albarello et al., Pratiques et méthodes de recherche en sciences sociales. Paris: Armand Colin.

Schubauer-Leoni, M.L., Leutenegger, F. \& Mercier, A. (1999). Interactions didactiques dans l'apprentissage des "grands nombres». In M. Gilly et al. (Ed.), Apprendre dans l'interaction, analyse des médiations sémiotiques. (pp. 301-328). Nancy et Aix en Provence: Presses Universitaires de Nancy \& Publications de l'Université de Provence.

Mots clés: conaissance, didactique, école primaire, enseignement, géographie, méthode d'enseignement 


\section{Primarlehrpersonen sprechen über Geografie und Geografieunterricht}

\section{Zusammenfassung}

Welche Lehrstoffe unterrichtet man gegenwärtig im Fach Geografie und wie geht man hier unterrichtlich vor? Obgleich man eigentlich eine sehr grosse unterrichtspraktische Vielfalt erwarten könnte, fördert die Analyse von halbdirektiven Interviews, die im Jahre 2001 mit sechs Französischwalliser Lehrpersonen der fünften und sechsten Primarklasse durchgeführt wurden, die Existenz von relativ fest etablierten unterrichtlichen Gewohnheiten zutage. Im Allgemeinen ist es ein verbessertes frontalunterrichtliches Vorgehen, das realisiert wird; das Wissen wird den Kindern gegenüber mehr oder weniger ausführlich verdeutlicht, bevor es institutionalisiert wird; die beschreibenden inhaltlichen Kenntnisse werden gegenüber dem praktischen Können und der Entwicklung von generellen intellektuellen Fähigkeiten bevorzugt, obschon man natürlich Letztere ebenfalls weiterentwickeln möchte; alle diese unterrichtspraktischen Gewohnheiten sind an eine bestimmte Vorstellung von Evaluation gebunden. Die disziplinären Traditionen werden in der vorliegenden Arbeit anhand der «mondes d'éducation» (nach Chatel), einem theoretischen Analyseinstrumentarium von einigen möglichen Erziehungskulturen untersucht, die sich im Schulzimmer im Zusammenspiel zwischen Lehrperson, Schülerinnen/Schülern und dem Wissen ergeben können.

Schlagworte: Didaktik, Geografie, Primarschule, Unterricht, Unterrichtsmethode, Wissen

\section{Insegnanti di scuola elementare parlano di geografia e del suo insegnamento}

\section{Riassunto}

Che cosa s'insegna attualmente a geografia e come si procede didatticamente? Se ci si potrebbe attendere una grande variazione nelle pratiche d'insegnamento, di fatto l'analisi di interviste semistrutturate con sei insegnanti vallesani di quinta e sesta classe elementare evidenziano delle abitudini ben strutturate. Normalmente si procede con un insegnamento frontale migliorato; i saperi vengono esplicitati lungamente; le conoscenze descrittive sono predominanti rispetto ai saper fare che comunque si vorrebbero sviluppare; all'origine di queste convergenze pratiche vi è in parte una determinata concezione della valutazione. Queste tradizioni d'insegnamento del sapere disciplinare vengono analizzate utilizzando i «mondes d'éducation» (Chatel 1997), uno strumento d'analisi dell'azione educativa che si instaura nel rapporto tra insegnante, allievi e sapere.

Parole chiave: conoscenza, didattica, geografia, insegnamento, metodo di insegnamento, scuola primaria 


\section{Primary school teachers speak about geography and how they teach it}

\section{Summary}

Which topics are currently taught in geography, and how do teachers proceed in teaching them? One would expect a large variety of teaching practices and methods. 6 primary school teachers, teaching geography for students in 5 th and 6 th grade in schools located in the French part of the Kanton of Wallis, Switzerland, were interviewed about this question. The analyses of these semi-standardized interviews revealed that the most frequently pursued method is some kind of optimized direct teaching, and is a quite stable teaching habit. Teachers believe to transfer their knowledge to the students and seem to promote descriptive content knowledge rather than practical skills and the development of general intellectual activities. The mentioned teaching habits correspond with the preference of a certain type of evaluation. "Mondes d'éducation", a theoretical frame of analyses by Chatel, is applied in order to investigate the tradition of educational cultures within the discipline. Educational cultures, on their part, are created in an interplay between teachers, students, and knowledge.

Key words: didactics, geography, knowledge, primary school, teaching, teaching method 